\title{
Citología Vaginal en Mujeres menores de 25 años
}

\author{
Dr. Hernando Navarro
}

\section{MOTIVACION:}

Evaluación de diez años de citologías, en búsqueda de la prevalencia de patología cervical en un grupo específico de mujeres.

\section{OBJETIVO:}

Evaluar la necesidad de citología vaginal en mujeres jóvenes y determinar una edad mínima para la toma de citología vaginal. Secundariamente, buscar factores de riesgo en este tipo de pacientes.

\section{INTRODUCCION}

De todos es conocido el incremento de los casos de carcinoma en el mundo y la búsqueda de soluciones mediante ingentes esfuerzos médicos; uno de los problemas graves de este tipo de patología es su diagnóstico tardío y el consecuente aumento de morbimortalidad. En la búsqueda de tratamientos curativos se hace necesario el diagnóstico precoz, factible en un buen número de casos, y especialmente en el carcinoma de cérvix, gracias a la aparición y desarrollo de la citología vaginal. A pesar de ello, en nuestro me- dio aún es frecuente la consulta tardía en carcinoma de cérvix uterino, por múltiples factores; por ello las entidades de salud, en forma continuada, en los últimos años, se han dedicado de lleno a la prevención de la enfermedad mediante campañas educativas y de servicio. Surge entonces una pregunta importante: ¿a qué edad se debe tomar la citología vaginal?. Existen estudios de citología vaginal en mujeres jóvenes en otros países, con datos sobre citología anormal y $\mathrm{Ca}$. de cérvix (10-11-12). En nuestro medio no hay un estudio que sirva de base para establecer tal correlación. Para tal efecto se tomó arbitrariamente la edad de 25 años para efectuar el análisis durante los diez años, comprendidos entre enero de 1970 y diciembre de 1979.

\section{METODOLOGIA.}

Sirvieron de base para el estudio las historias de los archivos del Programa de Cáncer de los Servicios de Salud del Municipio de Cali, tomando el grupo de mujeres menores de 25 años. Se analizaron las siguientes variables: edad, resultado de la citología, biopsias tomadas y su resultado, así como el seguimiento posterior, si lo hubo. 


\section{RESULTADOS}

En la década revisada se practicaron 326.450 citologías, sin hacer discriminación por edad, encontrándose 3.469 anormales, que representan el $1.06 \%$, cifra inquietante, siendo el $0.45 \%$ de casos de citologías IV y V (Cuadro 1). Sin. embargo, estos hallazgos guardan proporción estrecha con los encontrados en mujeres menores de 25 años (Cuadro 2). Se encontraron en esta década 35.000 citologías pertenecientes a mujeres de este grupo de edad, menores de 25 años, lo cual representa el $10.72 \%$ del total de citologías; con citologías anormales 294 pacientes que representan, en este grupo de menores de 25 años, el $0.84 \%$. Con citologías IV y $\mathrm{V}$ sólo el $0.15 \%$ que sí marca una diferencia con el grupo global mostrado en el cuadro 1, pero que para efectos de incidencia de cáncer en grupos de jóvenes es preocupante.

Cuadro No. 1

DISTRIBUCION CITOLOGIAS TOMADAS EN EL PERIODO 1970 - 1979

\begin{tabular}{|c|c|c|c|c|}
\hline Negativas & $\begin{array}{c}\text { Grado } \\
\text { III }\end{array}$ & $\begin{array}{c}\text { Grado } \\
\text { IV }\end{array}$ & $\begin{array}{c}\text { Grado } \\
\text { V }\end{array}$ & Total \\
\hline 322.982 & 1993 & 490 & 986 & 326.450 \\
\hline
\end{tabular}

Cuadro No. 2

\section{CITOLOGIAS ANORMALES MUJERES MENORES DE 25 AÑOS}

\begin{tabular}{|l|c|c|c|}
\hline Negativas & Grado III & Grado IV & Grado V \\
\hline 35.000 & 240 & 31 & 23 \\
\hline
\end{tabular}

A los 294 casos de citología anormal en menores de 25 años se hace un análisis más detallado en lo referente a los posibles factores que se dice juegan papel en la citología del $\mathrm{Ca}$. de cérvix. Cabe anotar que esta información fue extractada de la consignada en todos los casos. Se aprecia en el Cuadro 3 la distribución según el grado III, IV, V; se puede ver como no hay ningún caso de citología sospechosa de Ca. (IV o V) por debajo de 18 años, lo cual está de acuerdo con otras revisiones en nuestro medio (6), y contrasta con publicaciones de otros países ${ }^{8-9}$, en donde sí existe esta patología en edades menores de 18 años.

Cuadro No. 3

CITOLOGIAS ANORMALES DISTRIBUCION SEGUN SU EDAD

\begin{tabular}{|r|r|r|r|r|}
\hline & $\leqslant 15$ & $16-18$ & $19-21$ & $\geqslant 22$ \\
\cline { 2 - 5 } Citología III & 1 & 17 & 64 & 158 \\
Citología IV & 0 & 0 & 4 & 27 \\
Citología V & 0 & 0 & 5 & $18-$ \\
\hline
\end{tabular}

En los cuadros 4 a 8 se discriminan 240 casos de citología III en menores de 25 años. Desafortunadamente datos importantes, según la mayoría de los autores ${ }^{1-2-4}$, como son: paridad, inicio de actividad sexual, etc., no se encuentran sino en un número bajo de historias; ( 54 y 53 respectivamente); igual sucede con el seguimiento. De todos es conocido que en la citología III se hace imperativa la biopsia, y según el resultado, el seguiguimiento posterior, cosa que evidentemente no se cumplió en la mayoría de los casos, pues solo se registra entre 34 pacientes de los 240 casos. (Cuadro 7). En este grupo de 34 pacientes con citología III y que recibieron control se halló una citología $\mathrm{V}$ perteneciente a una paciente mayor de 18 años.

Los cuadros 9 al 12 hacen correlación similar para las citologías IV, y son igualmente válidas las críticas respecto a la 
carencia de información, a pesar de ser un número relativamente bajo de casos.

Cuadro No. 4

\section{DISTRIBUCION POR EDAD CITOLOGIA III}

\begin{tabular}{|c|r|r|r|r|}
\hline Grupo Edad & 15 & $16-18$ & $19-21$ & $22-25$ \\
\hline No. Casos & 1 & 17 & 64 & 158 \\
\hline
\end{tabular}

Cuadro No. 5

DISTRIBUCION POR PARIDAD

CITOLOGIA III

\begin{tabular}{|c|c|c|c|c|c|}
\hline No. de Hijos & 0 & 1 & 2 & 3 & 4 \\
\hline No. de Casos & 7 & 19 & 13 & 10 & 5 \\
\hline
\end{tabular}

Cuadro No. 6

INICIO DE ACTIVIDAD SEXUAL CITOLOGIA III

\begin{tabular}{|l|r|r|r|r|}
\hline Grupo Edad & 15 & $16-18$ & $19-21$ & $22-25$ \\
\hline No. de Pacientes & 4 & 35 & 13 & 1 \\
\hline
\end{tabular}

Cuadro No. 7

HALLAZGOS DE SEGUIMIENTO

CITOLOGIA III

\begin{tabular}{|l|c|c|c|c|c|}
\hline $\begin{array}{l}\text { Grado de Citología } \\
\text { Encontrado }\end{array}$ & I & II & III & IV & V \\
\hline No. de pacientes & 2 & 20 & 10 & 1 & 1 \\
\hline
\end{tabular}

Los cuadros 13 al 16 muestran los resultados de la citología $V$. En este grupo llama la atención primero, que las pacientes pertenecen a grupos de 19 o
Cuadro No. 8

HALLAZGOS DE BIOPSIA CITOLOGIA III

\begin{tabular}{|l|c|c|c|c|c|c|}
\hline Resultado Biopsia & N & Cerv. & DL & DM & DS & Ca.I.S. \\
\hline No. Pacientes & 10 & 31 & 36 & 20 & 9 & 7 \\
\hline
\end{tabular}

más años de edad; segundo, todas tienen antecedentes de paridad (uno o más) y tercero, una marcada tendencia a la iniciación temprana de relación sexual (de 18 o menos años).

Cuadro No. 9

DISTRIBUCION POR EDAD

CITOLOGIA IV

\begin{tabular}{|l|c|c|c|c|}
\hline Grupo de Edad & $\leqslant 15$ & $16-18$ & $19-21$ & $\geqslant 22$ \\
\hline No. de Casos & 0 & 0 & 4 & 27 \\
\hline
\end{tabular}

Cuadro No. 10

DISTRIBUCION POR PARIDAD

CITOLOGIA IV

\begin{tabular}{|l|l|l|l|l|l|}
\hline No. de Hijos & 0 & 1 & 2 & 3 & 4 \\
\hline No. de Casos & - & 2 & 3 & 2 & 0 \\
\hline
\end{tabular}

Cuadro No. 11

INICIO DE ACTIVIDAD SEXUAL

CITOLOGIA IV

\begin{tabular}{|c|c|c|c|c|}
\hline Grupos de Edad & $\leqslant 15$ & $16-18$ & $19-21$ & $\geqslant 22$ \\
\hline No. de Pacientes & 1 & 2 & 3 & 1 \\
\hline
\end{tabular}


Cuadro No. 12

HALLAZGOS DE BIOPSIAS

CITOLOGIA IV

\begin{tabular}{|l|c|c|c|c|c|}
\hline Resultado Biopsias & Cerv. & DL & DM & DS & $\begin{array}{c}\text { Ca.in } \\
\text { Situ }\end{array}$ \\
\hline No. de Pacientes & 1 & 5 & 4 & 4 & 7 \\
\hline
\end{tabular}

Cuadro No. 13

DISTRIEUCION POR GRUPOS DE EDAD CITOLOGIA $V$

\begin{tabular}{|l|r|r|r|r|}
\hline Grupos de Edad & $\leqslant 15$ & $16-18$ & $19-21$ & $\geqslant 22$ \\
\hline No. de Casos & 0 & 0 & 5 & 18 \\
\hline
\end{tabular}

Cuadro No. 14

DISTRIBUCION POR PARIDAD

CITOLOGIA $V$

\begin{tabular}{|l|l|l|l|l|r|}
\hline No. de Hijos & 0 & 1 & 2 & 3 & $\geqslant 4$ \\
\hline No. de Casos & 0 & 1 & 4 & 1 & 3 \\
\hline
\end{tabular}

Cuadro No. 15

INICIO DE ACTIVIDAD SEXUAL CITOLOGIA $V$

\begin{tabular}{|l|c|c|c|c|}
\hline Edad & $\leqslant 15$ & $16-18$ & $19-21$ & $\geqslant 22$ \\
\hline No. Pacientes & 5 & 3 & 1 & 0 \\
\hline
\end{tabular}

Cuadro No. 16

HALLAZGOS DE BIOPSIAS CITOLOGIA $V$

\begin{tabular}{|l|c|c|c|}
\hline Patologia & CERVICITIS & DS. & CA.IN SITU \\
\hline No. Pacientes & 2 & 4 & 7 \\
\hline
\end{tabular}

\section{COMENTARIOS}

Muchos datos sobre evolución, antecedentes, etc., no figuran en la mayoría de las historias, lo cual hace difícil presentar conclusiones; pero sí se puede aventurar un mínimo de recomendaciones, entre ellas, la determinación de una edad crítica para la toma de la citología vaginal.

A pesar de lo anterior, y aunque haría falta una revisión que incluya los casos normales $y$ todo el seguimiento adecuado para efectuar comparaciones, creo que el trabajo logra un objetivo importante, como es el de saber que el carcinoma de cérvix en Cali sí se presenta en un número no despreciable de pacientes jóvenes, en edades comprendidas entre los 18 y los 25 años. Igualmente, es notable que la mayoría de las pacientes con citología anormal tiene historia de inicio de vicia sexual temprana, igual que su vida reproductiva. Lo anterior está de acuerdo con estudios efectuados en otras partes. Todos estos hallazgos nos hacen creer que este grupo de pacientes, a pesar de su temprana edad debería tener un control especial. Es decir, la precocidad en el inicio de la actividad sexual $y$ reproductiva sería un factor de riesgo mayor para pacientes de menos de 20 años y a ellas debería tomárseles citología vaginal por lo menos una vez al año. Sería igualmente recomendable que toda paciente de 20 o más años tenga citología vaginal anual, en nuestro medio. 


\section{BIBLIOGRAFIA}

1. GUZMAN, N., BUENO, M. Tendencias del Carcinoma de Cérvix en Cali. Antioquia Médica. 24:249, 1974.

2. BUENO, Miguel. Carcinoma del Cérvix Uterino. Aspectos para su diagnóstico y tratamiento. Acta Médica del Valle 6:93, 1975.

3. BUENO, M. Detección precoz del Cáncer del Cuello Uterino. Primer Simposio Latinoamericano de Detección Precoz de Enfermedad. Cali, Colombia. Feb. 15-18, 1976. Oscar Echeverry, Ed. Universidad del Valle.

4. BUENO, M., ZUÑIGA, J. Evolución Citológica en 200 usuarias de anticonceptivos. IX Congreso Colombiano de Obstetricia y Ginecología. Bucaramanga, Dic. 1971.

5. Bueno, M. Cáncer del Cérvix Uterino. Aspectos Epidemiológicos. X Congreso Colombiano de Ginecología y Obstetricia. Medellín, Dic. 1977.

6. ZUÑIGA, J., MONTAÑO, E. Observaciones sobre Citología Vaginal, Biopsia y Conización Cervical en Displasia y $\mathrm{Ca}$. in Situ de Cérvix. Comunicación Personal.

7. FELDMAN, M.J., LINZCY, E.M., SPEBNIK, E., KENTE, D.R., GOLDSTEIN, A., NELSON, M. Abnormal Cervical Citology in the teen agers. A continuing problem. Am. J. Obstet. Gynecol. 126: 418-21, Oct. 15, 1976.

8. FERGUNSON, J. H. Positive Cancer Smears in teen agers. J.A.M.A., 178 365, 1961.

9. KAUFMAN, R.H., BURMEISER, R.E. and SPJUT, H.J. Cervical Cytology in the teen age patient. Am. J. Obstet. Gynecol. 108-515.

10. RICHARD, K.M. and BARROW, B.A. A fallow-up study of patients with cervical dysplasia. Am. J. Obstet. Gynecol. 105: 386, 1969.

11. GONDONS, B., TOWNSEND, D.E. and Ostergard, D.R. Citologic diagnosis of squamous dysplasia and carcinoma of the cervix. Am. J. Obstet. Gynec. 110: 107. 11. 1971.

12. SEDLIS, M., COHEN, A. and SALL, S. The Fate of Cervical Dysplasia. Am. J. Obstet. Gynec. 107: 1065, 1970. 